\title{
Politique
}

\section{Les journalistes des quotidiens québécois et leur métier}

\section{Simon Langlois et Florian Sauvageau}

Volume 1, numéro 2, automne 1982

Les médias et les pouvoirs

URI : https://id.erudit.org/iderudit/040400ar

DOI : https://doi.org/10.7202/040400ar

Aller au sommaire du numéro

Éditeur(s)

Société québécoise de science politique

ISSN

0711-608X (numérique)

Découvrir la revue

Citer cet article

Langlois, S. \& Sauvageau, F. (1982). Les journalistes des quotidiens québécois et leur métier. Politique, 1(2), 5-39. https://doi.org/10.7202/040400ar d'utilisation que vous pouvez consulter en ligne.

https://apropos.erudit.org/fr/usagers/politique-dutilisation/ 


\title{
Les journalistes des quotidiens québécois et leur métier*
}

\author{
Simon Langlois \\ Université Laval \\ Florian Sauvageau \\ Université Laval
}

Les enjeux politiques, les questions d'idéologies et de structures, de propriété des entreprises et de contrôle de l'information ont dominé les débats sur les médias au Québec depuis une quinzaine d'années. Quelques chercheurs (certains dans le giron de l'Institut Canadien d'Éducation des Adultes ou des centrales syndicales: CSN, 1973 et CEQ-ICEA, 1979) ont publié divers travaux dénonçant l'influence des patrons de presse sur les contenus, et partant des pouvoirs économiques et de leurs alliés politiques. ${ }^{1}$ Pour d'autres observateurs au contraire, les nombreuses grèves des journalistes qui ont marqué cette période, en particulier celles dont les enjeux principaux touchaient au pouvoir dans les salles de rédaction, ont

\footnotetext{
* Nous remercions la Commission Royale sur les quotidiens de nous avoir permis d'utiliser les données d'un sondage effectué à son intention. Nous remercions aussi, pour leurs commentaires pertinents, Jean De Bonville et Gilles Lesage qui ont lu la première version de ce texte. Notre analyse n'engage cependant ni la Commission ni MM. Lesage et De Bonville.

1. CSN, La grande tricherie, Montréal, 1973, 272 p.; CEQ-ICEA, La parole ça se prend, Rapport et dossier de référence du colloque populaire sur le rôle des médias, leur accessibilité, leur contrôle et leur propriété, Montréal, 1979, 116 p.
} 
contribué à donner du journaliste l'image d'un radical cherchant à contrôler l'information. Cependant, l'on compte encore peu de travaux consacrés aux journalistes eux-mêmes et à leurs pratiques professionnelles. L'enquête la plus complète à ce sujet est sans doute celle qu'a dirigée Roger de La Garde en 1972 et 1974 dont seules les données sociographiques ont été publiées $^{2}$. Pierre Godin a aussi tracé un portrait-robot des journalistes québécois, à partir d'une enquête auprès des journalistes de La Presse, du Devoir, du Soleil et de Radio-Canada. Il a toutefois négligé d'interroger les journalistes de la presse populaire que l'on peut difficilement oublier quand on sait l'importance prise par ces quotidiens depuis dix ans. À Montréal, la part du marché des quotidiens de langue française détenue par Le Journal de Montréal est passée de $16 . \%$ en 1970 à $61.7 \%$ en $1980 .^{3}$ Certains chercheurs se sont aussi intéressés à des aspects particuliers du journalisme ou encore au journalisme d'une région donnée, mais dans l'ensemble on sait peu de choses des artisans de l'information. ${ }^{4}$

2. De La Garde, Roger, «Profil socio-démographique des journalistes de la presse écrite québécoise», Communication et information, 1, 1, 1975: 31-52; De La Garde, Roger et Bernard Barrett, "Profil socio-démographique des journalistes de la presse électronique québécoise», Communication et information, 1, 3, automne 1976: 259-279.

3. Commission Royale sur les quotidiens, Les quotidiens et leurs lecteurs, Publications de recherches, Volume 1, Ottawa, 1981, tableau 88, 100.

4. Citons l'étude de Jean De Bonville sur les sources d'information des journalistes de cinq quotidiens québécois, les travaux de Jacques Benjamin, «Les journalistes face au pouvoir politique québécois depuis 1960: une approche sociétale», Communication présentée au Congrès annuel de l'Association Canadienne de Recherche Sociale Appliquée, Université du Québec à Montréal, 4 juin 1980, 28 p. «Pouvoir politique et médias au Québec», Communication et information, 3, 1, 1979: 6777; l'enquête de Marcel Gilbert, "L'information gouvernementale et les courriéristes parlementaires au Québec», Revue Canadienne de Science Politique, 4, 1, mars 1971: 26-51; Les recherches du CRIDEQ, Lévesque, B., Jean Larrivée et C. Morin, Les entreprises de mass média de l'Est du Québec et leur personnel (enquête auprès des patrons), Rimouski, Université du Québec à Rimouski, 1978, 1380. (cahiers du CRIDEQ); Lévesque, B. et J. Larrivée, Sociographie du personnel spécialisé des mass média de l'Est du Québec, Rimouski, Université du Québec à Rimouski, 1979, 210 p. (cahiers du CRIDEQ); L'ouvrage collectif édité par Sauvageau, Florian, Gilles Lesage et J. De 


\section{TABLEAU 1}

Tirage des quotidiens québécois de langue française en septembre 1980 et nombre de journalistes à temps plein en janvier 1981

\begin{tabular}{lccc}
\hline & $\begin{array}{c}\text { Tirage } \\
\text { en semaine }\end{array}$ & $\begin{array}{c}\text { Tirage } \\
\text { le samedi }\end{array}$ & $\begin{array}{c}\text { Nombre de } \\
\text { journalistes }\end{array}$ \\
\hline $\begin{array}{l}\text { Le Journal de Montréal } \\
\text { La Presse }\end{array}$ & 314,698 & 305,213 & 69 \\
Le Soleil & 185,000 & 283,582 & 125 \\
Le Journal de Québec & 122,484 & 130,576 & 106 \\
Le Nouvelliste (Trois-Rivières) & 102,486 & 93,275 & 33 \\
La Tribune (Sherbrooke) & 50,839 & - & 40 \\
Le Devoir & 41,056 & 43,824 & 30 \\
Le Quotidien (Chicoutimi) & 40,732 & 40,503 & 38 \\
La Voix de l'Est (Granby) & 21,260 & - & 17 \\
\hline
\end{tabular}

Les tirages sont en date de septembre 1980, sauf pour La Presse (31-12-80) et ils sont tirés de Canadian Advertising Rates and Data.

Nous nous attarderons ici à dégager les attitudes et les orientations personnelles des journalistes de la presse écrite quotidienne francophone du Québec à l'aide des données d'un sondage effectué auprès d'un échantillon de journalistes à l'emploi des neuf quotidiens dont la liste apparaît au tableau $1 .{ }^{5}$ Il s'agit donc de voir ce que sont les journalistes à travers l'image qu'ils se donnent d'eux-mêmes. Après avoir identifié

Bonville, Les journalistes, Montréal, Éditions Québec-Amérique, 1980, 421 p.; Et plus récemment les travaux de recherche de la Commission Kent: Commission Royale sur les quotidiens, Rapport, Ottawa, Ministre des approvisionnements et services, 1981, 323 p.; Commission Royale sur les quotidiens, Les quotidiens et leurs lecteurs, Publications de recherches, vol. 1, Ottawa, 1981; Commission Royale sur les quotidiens, Les relations de travail dans l'industrie des quotidiens, Publications de recherches, vol. 5, Ottawa, 1981.

5. Ce sondage a été mené auprès de la moitié des 471 journalistes de la presse francophone au mois de février 1981. Le taux de réponses a été élevé (63.4\%) et l'échantillon est représentatif de la population visée. Le lecteur trouvera toutes les précisions habituelles et les renseignements sur les aspects méthodologiques dans l'annexe au volume 2 des Études sur l'industrie des quotidiens, publié par la Commission Royale d'enquête sur les quotidiens (Commission Kent). 
certaines caractéristiques personnelles des journalistes interrogés (scolarité, âge, expérience) nous ferons quelques commentaires sur le journalisme comme métier ou profession. Nous proposerons une typologie des journalistes afin de rendre compte de l'hétérogénéité des conceptions du métier, qu'il s'agisse de l'importance accordée à diverses fonctions ou des qualités requises pour être un bon journaliste. Nous nous demanderons ensuite si la pratique quotidienne du métier correspond à l'image que s'en font les journalistes et nous chercherons à voir jusqu'à quel point la nature des entreprises de presse intervient en même temps que les orientations personnelles des journalistes pour structurer la pratique de leur métier. Nous mesurerons enfin le jugement que les journalistes interrogés portent sur le journalisme pratiqué au Québec et nous verrons ce qu'ils pensent du traitement que réserve la presse aux divers acteurs sociaux.

\section{Un groupe hétérogène}

Les journalistes ne forment pas un groupe homogène. On n'arrive pas à ce métier à partir d'une seule formation qui servirait en quelque sorte de pørte d'entrée vers l'exercice d'une profession dont l'accès serait contrôlé. Au contraire, la formation des journalistes est diversifiée et une proportion importante d'entre eux n'ont pas poursuivi une scolarité poussée, principalement dans le groupe des employés de Québécor et dans les journaux régionaux. ${ }^{6}$ Ainsi, chez Québécor, la scolarité moyenne est de 14.8 années alors qu'elle s'élève à 16.1 années dans les trois quotidiens d'information grand format

6. Nous avons regroupé les entreprises de presse de la façon suivante: les quotidiens d'information grand format publiés à Québec et à Montréal (la Presse, Le Devoir, le Soleil), les journaux populaires format tabloid du groupe Québécor (Le Journal de Montréal et le Journal de Québec) et les quotidiens régionaux (La Voix de l'Est, la Tribune, le Nouvelliste, le Quotidien). 


\section{TABLEAU 2}

Diplôme le plus élevé détenu par les journalistes, selon le type de quotidiens

\begin{tabular}{|c|c|c|c|c|}
\hline Diplôme & $\begin{array}{l}\text { Quotidiens grand format } \\
\text { Montréal et Québec }\end{array}$ & $\begin{array}{l}\text { Quotidiens } \\
\text { Québécor }\end{array}$ & $\begin{array}{l}\text { Quotidiens } \\
\text { régionaux }\end{array}$ & Total \\
\hline - Secondaire et moins & 15.2 & 32.1 & 24.1 & 20.6 \\
\hline - Bacc. ès arts & 30.4 & 21.4 & 10.4 & 24.3 \\
\hline - D.E.C. & 1.3 & 21.4 & 24.1 & 10.3 \\
\hline $\begin{array}{l}\text { - Études universitaires } \\
\text { non complétées }\end{array}$ & 2.5 & 3.6 & 6.9 & 3.6 \\
\hline - Brevet d'enseignement & 3.8 & 3.6 & 6.9 & 4.4 \\
\hline$-1^{\mathrm{er}}$ cycle & 35.4 & 14.3 & 13.8 & 26.5 \\
\hline$-2^{\mathrm{e}}$ et $3^{\mathrm{e}}$ cycles & 11.4 & 3.6 & 6.9 & 8.9 \\
\hline - Autres & - & - & 6.9 & 1.4 \\
\hline Total & $\begin{array}{c}100 . \\
79\end{array}$ & $\begin{array}{c}100 . \\
28\end{array}$ & $\begin{array}{c}100 . \\
29\end{array}$ & $\begin{array}{l}100 . \\
136\end{array}$ \\
\hline
\end{tabular}

TABLEAU 3

Caractéristiques des journalistes (âge, scolarité, expérience, salaire) selon le type de quotidiens

\begin{tabular}{lcccc}
\hline & $\begin{array}{c}\text { Quotidiens grand format } \\
\text { Montréal et Québec }\end{array}$ & $\begin{array}{c}\text { Quotidiens } \\
\text { Québécor }\end{array}$ & $\begin{array}{c}\text { Quotidiens } \\
\text { régionaux }\end{array}$ & Total \\
\hline - Âge & 40.3 & 35.1 & 36.6 & 38.5 \\
- Scolarité & 16.1 & 14.8 & 15.5 & 15.7 \\
- Expérience totale & 15.9 & 10.9 & 11.2 & 13.9 \\
- Expérience dans & 11.8 & 5.5 & 9.1 & 9.9 \\
$\begin{array}{l}\text { le journal } \\
\text { - Salaire }\end{array}$ & $2761 \$$ & $31890 \$$ & $23636 \$ 27527 \$$ \\
\hline
\end{tabular}

de Québec et de Montréal (tableaux 2 et 3). Les diplômés d'universités sont plus nombreux dans ces derniers $(46.8 \%$ au total) et les personnes détenant une scolarité secondaire (complétée ou non) se retrouvent en plus fortes proportions à l'emploi des entreprises de presse régionales et dans le groupe Québécor. 
Fait à noter, les journaux grand format de Québec et de Montréal ont embauché il y a quelques années un nombre important de personnes qui avaient complété leur cours classique (Baccalauréat ès arts), mais une fois les collèges classiques disparus, tout se passe comme s'ils s'étaient plutôt tournés vers l'engagement de diplômés d'universités en ignorant les diplômés des Cégeps. Au contraire, les journaux régionaux et ceux qui font partie du groupe Québécor ont recruté plusieurs diplômés des Cégeps et beaucoup moins de diplômés d'universités.

L'examen d'autres indicateurs permet aussi de faire ressortir l'hétérogénéité des personnes qui exercent ce métier. Les journalistes à l'emploi des grands quotidiens de Québec et de Montréal se distinguent encore de leurs collègues des autres journaux. Ils sont plus âgés ( 40.3 ans en moyenne), ils ont davantage d'années d'expérience dans l'exercice de ce métier (15.9 ans) et ils sont à l'emploi de leur journal depuis plus longtemps (11.8 ans). Les journaux publiés par le groupe Québécor comptent des journalistes ayant en moyenne moins d'expérience (10.9 années), moins d'ancienneté dans le journal ( 5.5 ans) et la moyenne d'âges y est plus basse (35.1 ans). Les journalistes à l'emploi des grands quotidiens de Québec et de Montréal gagnaient en moyenne 27 461\$ en février 1981, contre $31890 \$$ pour ceux qui étaient à l'emploi des journaux du groupe Québécor. Il s'agit là d'un écart important, qui s'accentue encore si l'on tient compte du fait que ces derniers ont moins d'années d'expérience, que leur scolarité moyenne est moins élevée et qu'ils sont plus jeunes. ${ }^{7}$ Les caractéristiques des journalistes de la presse quotidienne régionale sont très

7. Nous avons demandé aux répondants d'indiquer leur salaire de base, en excluant le temps supplémentaire. En juillet 1982, les conventions collectives des quotidiens grand format et de la presse populaire laissant toujours voir des écarts importants dans les échelles de salaires. Il faut noter que les politique d'allocation de temps supplémentaires, qui varient d'une entreprise à l'autre, peuvent modifier ces écarts. 
proches de celles des journalistes du groupe Québécor, sauf pour ce qui est de l'ancienneté dans le journal, plus importante, et du salaire, qui était plus faible en moyenne (23 636\$).

Les journalistes de la presse quotidienne au Québec étaient en 1981 plus scolarisés et plus âgés en moyenne que ceux et celles qui exercaient ce métier dix ans plus tôt, comme en témoigne une comparaison de nos résultats avec ceux tirés de l'enquête de Roger de la Garde menée au cours de l'été 1972. ${ }^{8}$ Dans l'ensemble, la proportion des diplômés d'universités a augmenté de $25 . \%$ à $35 . \%$ entre 1972 et 1981 , celle des finissants des collèges classiques a quelque peu diminué, passant de $31 . \%$ à $24 . \%$ et celle des diplômés des Cégeps est passée de $4 . \%$ à $10 . \%$. Il semble enfin que la proportion des journalistes qui ne détiennent aucun diplôme ait diminué de moitié au cours de ces dix années. Roger de la Garde ne donne pas l'âge moyen des journalistes de la presse quotidienne qui ont participé à son enquête mais il présente toutefois une distribution de fréquences entre trois groupes d'âges. En 1972, 42\% des journalistes avaient moins de 30 ans. Cette proportion tombait à $12.8 \%$ en 1981. Dans le groupe d'âges 30-39, la proportion était de $36 . \%$ en 1972 et de $47 . \%$ dix ans plus tard et dans le groupe des journalistes ayant 40 ans ou plus, on note une augmentation de $23 . \%$ en 1972 à $40.2 \%$ en 1981. À la lumière de ces données, il est donc possible de conclure que les journalistes québécois sont maintenant plus scolarisés qu'il y a dix ans et qu'ils sont aussi plus âgés en moyenne. Compte tenu en particulier du fait que les journalistes ont maintenant environ 10 ans d'expérience dans le même journal (et même 11.8 ans dans les quotidiens grand format de Montréal et de Qué-

8. Cette comparaison entre nos données et celles de Roger De La Garde (1975) est présentée à titre indicatif car les deux échantillons ne sont pas strictement comparables. Nous n'avons retenu de l'enquête de cet auteur que les données relatives aux journalistes à l'emploi des quotidiens. 
bec), il est permis de faire l'hypothèse que la grande mobilité qui a pu caractériser les journalistes auparavant s'est résorbée, dans le cas de la presse quotidienne du moins.

Le recrutement en proportions encore élevées de personnes n'ayant acquis qu'une formation générale de base et la grande diversité des formations à l'intérieur de chacune des entreprises de presse indiquent clairement que le journalisme est un métier qui s'apprend sur le tas avant d'être une profession au sens sociologique du terme. Mais il faut surtout noter le rôle clé que joue l'entreprise, puisqu'elle est relativement libre d'engager qui elle veut et, partant, de définir ainsi le profil de ceux qui feront partie de la confrérie des journalistes. En d'autres termes, le profil des journalistes - et principalement leur formation - sont jusqu'à un certain point ajustés aux besoins de l'entreprise, qui engage en fonction de la définition qu'elle se donne du journalisme. Le journaliste n'est pas non plus un professionnel au sens classique du terme, qui exerce un large contrôle sur son propre travail. Les paramètres généraux du produit qu'il contribue à fabriquer (le journal) sont définis par d'autres et il jouit d'une autonomie limitée dans l'exercice quotidien de son métier parce que les sujets qu'il traite sont le plus souvent choisis en accord avec son supérieur. Le journaliste est un salarié qui doit négocier non seulement sa rémunération mais aussi les conditions d'exercice de son métier, ce qu'il est convenu d'appeler les clauses professionnelles. On sait de plus qu'à de rares exceptions près, les journalistes, même les plus préoccupés par les questions professionnelles ont toujours refusé d'assimiler leur tâche à celle d'un professionnel au sens traditionnel du terme, et se sont toujours opposés à l'idée d'une corporation professionnelle de journalistes, avec son profil de formation, des normes d'accès à la profession, etc. Les journalistes n'ont pas à acquérir une formation académique commune, formation qui contribue à définir et à 
légitimer l'existence d'une profession, mais ils ont toujours plaidé au contraire pour la diversité des formations et des expériences de vie du futur journaliste qui seule sera garante disent-ils - d'approches différentes dans la perception des événements et partant, du pluralisme de l'information. C'est sans doute ce qui explique que plusieurs journalistes n'aient pas vu d'un bon oil l'idée de dispenser une formation universitaire ou collégiale en journalisme.

\section{Les journalistes et leur conception du métier}

Au cours des années 60, on a vu se développer aux ÉtatsUnis une approche plus engagée du journalisme qui mettait l'accent sur l'interprétation de la nouvelle, le journaliste ne se satisfaisait plus du rôle de spectateur ou de simple reporter auquel l'avait confiné la presse d'information. Un mouvement semblable, qu'il faut distinguer du journalisme d'opinion, est aussi apparu en Europe. Un chercheur italien l'a fort bien décrit de la façon suivante: "Donner une interprétation, ce n'est pas tellement donner un commentaire à la nouvelle qu'être capable d'en donner une interprétation rationnelle, c'est-à-dire de la voir dans son contexte et dans la succession des événements qui l'ont précédée ou qui lui sont parallèles ${ }^{9}$. Cette orientation n'est rien d'autre que ce que la célèbre Commission Hutchins recommandait dès les années 40 dans son rapport devenu le classique en matière de responsabilité sociale des médias.

Dans une enquête menée auprès des journalistes américains, Johnstone et ses collaborateurs (1976) distinguent deux attitudes différentes des journalistes vis-à-vis de leur métier, en opposant ceux qui proposent un journalisme neutre et ceux

9. Bechelloni, Giovanni, "Quel type de journaliste et quel type de journalisme», Études de radio-télévision, Publications R.T.B.F., Bruxelles, 27, mai 1980, 171 et suivantes. 
qui affectionnent un journalisme participant. Selon la première attitude, le journaliste se doit de transmettre au public une information qui colle le plus possible aux faits qu'il rapporte avec tout le détachement dont il est capable en prenant soin de bien vérifier ses sources et l'exactitude des informations. Le journaliste est alors le rapporteur fidèle des faits les plus divers, rôle que Johnstone identifie à celui du spectateur. L'autre attitude exige du journaliste un plus grand engagement, prenant pour acquis que l'information brute et neutre n'existe pas, car elle trahit toujours une prise de position de la part de celui qui la transmet. Le journaliste se voit ici confier un rôle plus actif, plus «créateur». Il doit mettre en opposition diverses sources, recouper les faits afin de faire ressortir des enjeux. L'attitude participante consiste à situer les informations dans une perspective précise ou dans un contexte donné dont le journaliste doit tenir compte.

a) Une typologie des journalistes

En nous inspirant de cette opposition entre deux grands courants, nous proposons de construire une typologie des journalistes qui semble appropriée pour rendre compte de la diversité des conceptions de la pratique du métier. Nous distinguerons quatre types de journalistes:

1. Le premier type comprend les journalistes qui cherchent à convaincre et qui se situent dans la tradition du journalisme d'opinion. Ce journaliste se présentera souvent comme un agent de changement ou de développement. Nous l'appellerons l'éducateur;

2. vient ensuite l'enquêteur ou l'analyste qui désire jouer un rôle actif dans la transmission de l'information mais en limitant son travail aux faits, soit en les mettant en contexte soit en cherchant à débusquer le fait caché derrière l'événement; 
3. le troisième type de journaliste, le reporter, privilégie la relation des faits tels qu'ils lui sont présentés et son attitude est plus passive;

4. enfin, le dernier type se préoccupe surtout d'intéresser le lecteur; il veut vendre son produit et il se soucie de l'emballage. Nous le qualifierons de séducteur.

Cette typologie, qui n'a rien de rigide, puisque l'enquêteur peut aussi vouloir intéresser le lecteur et que le reporter peut parfois provoquer le changement, se différencie de certaines autres. Ainsi, G. Grant distingue plutôt trois types de journalistes:

1. le reporter, hautement dépendant de sources officielles, la "courroie de transmission», dit Grand; le haut-parleur des divers pouvoirs, pourrions-nous dire;

2. l'enquêteur, qui travaille à partir de sources personnelles, auxquelles il fait entière confiance;

3. l'analyste, plus «intellectuel», dont les méthodes de travail rejoignent, à la limite, celles du spécialiste des sciences sociales ${ }^{10}$.

Notre typologie nous semble mieux refléter la diversité du milieu journalistique québécois. Elle tient à la fois compte de la presse populaire qui a pris beaucoup de place depuis quinze ans et de la situation particulière du Québec où la pratique journalistique s'inspire à la fois de la tradition nordaméricaine fondée sur le fait (sacré) et de la tradition européenne du journalisme d'opinion. Nous avons aussi réuni sous une même étiquette l'enquêteur et l'analyste que Grant distingue, compte tenu du désir commun de l'un et de l'autre d'aller au-delà de l'image que les divers acteurs sociaux veulent donner

10. Grant, G., "Journalism and social sciences: continuities and discontinuities », dans H. Gans (ed), On the making of Americans, University of Pennsylvania Press, 1979: 291-313. 
TABLEAU 4

Opinion des journalistes portant sur diverses fonctions qu'un journaliste devrait remplir

\begin{tabular}{|c|c|c|c|c|c|c|}
\hline & & $\begin{array}{l}\text { très } \\
\text { important }\end{array}$ & $\begin{array}{c}\text { assez } \\
\text { important }\end{array}$ & $\begin{array}{l}\text { peu } \\
\text { important }\end{array}$ & $\begin{array}{l}\text { pas } \\
\text { important }\end{array}$ & $\begin{array}{c}\text { Total } \\
\mathrm{N}=149\end{array}$ \\
\hline 1 & $\begin{array}{l}\text { Faire enquête sur les activités des } \\
\text { gouvernements et organisations publiques }\end{array}$ & 59.7 & 36.3 & 4. & - & 100. \\
\hline 2 & $\begin{array}{l}\text { Être un agent de changement dans le } \\
\text { milieu }\end{array}$ & 23.6 & 41.9 & 28.4 & 6.1 & 100. \\
\hline 3 & $\begin{array}{l}\text { Transmettre l'information le plus } \\
\text { rapidement possible }\end{array}$ & 59.1 & 32.9 & 7.3 & 0.7 & 100 \\
\hline 4 & $\begin{array}{l}\text { Analyser et interpréter les enjeux } \\
\text { difficiles }\end{array}$ & 57.1 & 35.4 & 6.8 & 0.7 & 100 \\
\hline 5 & $\begin{array}{l}\text { Rapporter fidèlement les propos des } \\
\text { personnalités rencontrées }\end{array}$ & 76.4 & 19.6 & 4. & - & 100 \\
\hline 6 & $\begin{array}{l}\text { Publier les nouvelles qui intéressent } \\
\text { le plus grand nombre de lecteurs }\end{array}$ & 52.7 & 35.8 & 10.8 & 0.7 & 100 \\
\hline 7 & $\begin{array}{l}\text { Étendre le champ des préoccupations } \\
\text { culturelles et intellectuelles du } \\
\text { public }\end{array}$ & 41.5 & 43.5 & 13.6 & 1.4 & 100. \\
\hline 8 & Meubler les loisirs du public, divertir & 13.6 & 40.8 & 33.3 & 12.3 & 100. \\
\hline
\end{tabular}


et du message officiel qu'ils cherchent à faire passer. Les moyens utilisés peuvent varier, mais dans les deux cas, l'intention est la même.

Opératoirement, la typologie a été construite de la façon suivante. Nous avons d'abord demandé aux journalistes de donner leur opinion sur l'importance qu'ils accordaient à diverses fonctions dans l'exercice de leur métier. Les réponses à ces questions apparaissent dans le tableau 4. Nous avons ensuite proposé aux répondants d'identifier la fonction qui leur semblait la plus importante. Ces réponses ont permis de construire une typologie empirique des journalistes à partir de leur autoclassement - et non à partir d'une analyse des relations entre les questions elles-mêmes. ${ }^{11}$

Les journalistes québécois de la presse écrite francophone se répartissent assez bien entre ces quatre types (tableau 5).

\section{TABLEAU 5}

Typologie des journalistes de la presse quotidienne selon le type de quotidiens

\begin{tabular}{lcccc}
\hline $\begin{array}{l}\text { Types de } \\
\text { journalistes }\end{array}$ & $\begin{array}{c}\text { Quotidiens grand format } \\
\text { Montréal et Québec }\end{array}$ & $\begin{array}{c}\text { Quotidiens } \\
\text { Québécor }\end{array}$ & $\begin{array}{c}\text { Quotidiens } \\
\text { régionaux }\end{array}$ & Total \\
\hline $\begin{array}{l}\text { éducateur } \\
\text { enquêteur- }\end{array}$ & 24.7 & 10. & 23.3 & 21.4 \\
$\begin{array}{l}\text { analyste } \\
\text { reporter }\end{array}$ & 38.8 & 20. & 20. & 31. \\
séducteur & 27.1 & 36.7 & 43.3 & 32.4 \\
\hline Total \% & 9.4 & 33.3 & 13.4 & 15.2 \\
\hline \multicolumn{1}{c}{$\mathrm{N}$} & 100. & 100. & 100. & 100. \\
\hline
\end{tabular}

$\mathrm{p}=.01$

11. Les fonctions 2 et 7 identifient l'éducateur, les fonctions 1 et 4 , l'enquêteur-analyste, les fonctions 3 et 5 , le reporter et enfin les fonctions 6 et 8 , le séducteur. L'association entre les items est satisfaisante: les coefficients gamma sont de $.39, .48,-.39$ et .51 . Notons que la validité pragmatique de la typologie est bonne. Cette mesure permet très bien de discriminer les répondants lorsqu'on la met en relation avec diverses variables. 
L'enquêteur et le reporter comptent pour $31 \%$ et $32.4 \%$ des répondants, l'éducateur, $21.4 \%$ et le séducteur, $15.2 \%$. Au total, il n'y a donc pas de type vraiment dominant. Il en va autrement lorsqu'on isole les catégories de journaux puisque les types de journalistes que nous avons distingués se distribuent très différemment selon la nature des journaux. La proportion des enquêteurs-analystes est plus élevée dans les quotidiens à grand format de Québec et de Montréal (Le Soleil, La Presse et Le Devoir). C'est dans la presse quotidienne régionale qu'on retrouve la plus forte proportion de journalistes qui se définissent comme reporters. Le tiers des journalistes à l'emploi du groupe Québécor se définissent comme séducteurs, cherchant avant tout à intéresser le lecteur, cette proportion des séducteurs étant environ trois fois plus élevée que celle qui a été observée dans les autres sortes de quotidiens. Enfin, le type de l'éducateur est sous-représenté dans les journaux du groupe Québécor et il compte pour le quart des journalistes dans les autres quotidiens.

Les quatre types de journalistes que nous avons définis se distinguent selon certaines caractéristiques personnelles (tableau 6). Les reporters sont un peu plus âgés et ils comptent davantage d'années d'expérience dans la pratique du journalisme. Les séducteurs sont plus jeunes et ils ont moins d'expérience. Les éducateurs ont un profil qui les rapproche des enquêteursanalystes. Enfin, les quatre types de journalistes se différencient assez peu quant à leur scolarité : les éducateurs ont tendance à être plus scolarisés mais cette relation n'est pas statistiquement significative.

Dans l'ensemble, la façon de voir le métier de journaliste varie selon certains traits personnels des répondants mais aussi selon la nature des entreprises qui les emploient. L'appartenance à un type donné d'entreprise de presse a-t-elle plus ou moins d'importance que les traits personnels dans la conception que 
TABLEAU 6

Typologie des journalistes de la presse quotidienne selon l'âge, la scolarité et l'expérience dans le journalisme (en moyennes)

\begin{tabular}{lccc}
\hline \multicolumn{1}{c}{ types } & scolarité & âge & $\begin{array}{c}\text { expérience dans le } \\
\text { journalisme }\end{array}$ \\
\hline éducateur & 16.3 & 38.6 & 14.6 \\
enquêteur- & 15.9 & 37.7 & 13.6 \\
analyste & 15.4 & 40.9 & 15.5 \\
reporter & 15.2 & 35.7 & 10.9 \\
séducteur & 15.7 & 38.6 & 14. \\
\hline Total & & & \\
\hline
\end{tabular}

les journalistes se font de leur métier? C'est ce que nous tenterons de voir en adoptant une méthodologie qui permet de considérer en même temps plusieurs facteurs.

\section{b) L'orientation professionnelle des journalistes}

Après avoir analysé la façon dont les journalistes définissent eux-mêmes la fonction qu'ils jugent la plus importante dans l'exercice de leur métier, nous tenterons de préciser leur attitude professionnelle en construisant deux échelles, l'une mesurant l'orientation vers la participation et l'autre, l'orientation vers la neutralité, échelles qui s'inspirent des travaux de Johnstone et alii déjà cités. Ces échelles ne répartissent plus les individus en catégories nominales, comme c'est le cas dans une typologie, mais elles permettent de leur donner une position sur un continuum en indiquant s'ils sont plus ou moins favorables à la neutralité ou s'ils sont plus ou moins sympathiques à un journalisme plus intervenant et critique. ${ }^{12}$ L'intérêt de

12. Les deux échelles de type Likert ont été construites à partir des items apparaissant dans le tableau 4. Les indicateurs 1,4 et 7 sont entrés dans la construction de l'échelle orientation vers la participation et les indicateurs 3, 6 et 8 , dans la 
cette construction n'est pas d'en arriver à une estimation du nombre de journalistes participants ou neutres. Il paraît au contraire préférable de dégager les facteurs qui provoquent telle ou telle orientation. Au moyen d'une analyse de régression sur les deux échelles, nous serons en mesure de considérer en même temps sept variables dans le but de répondre à la question posée plus haut: jusqu'à quel point le type d'entreprise de presse affecte-t-il - indépendamment des caractéristiques des individus - les façons de voir la pratique du journalisme? Le choix des variables a été effectué afin d'inclure dans le modèle non seulement des caractéristiques individuelles (scolarité, âge, expérience dans le journalisme), mais aussi des mesures du statut dans l'entreprise (salaire, position occupée, syndicalisation) et une variable structurelle (le type d'entreprise).

TABLEAU 7

Régression d'un ensemble de variables sur l'échẹlle de participation et l'échelle de neutralité

\begin{tabular}{|c|c|c|c|c|}
\hline \multirow[b]{2}{*}{ Variables } & \multicolumn{2}{|c|}{ Participation } & \multicolumn{2}{|c|}{ Neutralité } \\
\hline & $\begin{array}{l}\text { Coefficient } \\
\text { de régression }\end{array}$ & $\begin{array}{c}\text { Coefficient } \\
\text { de corrélation }\end{array}$ & $\begin{array}{l}\text { Coefficient } \\
\text { de régression }\end{array}$ & $\begin{array}{c}\text { Coefficient } \\
\text { de corrélation }\end{array}$ \\
\hline scolarité & 07 & .09 & $-.23^{*}$ & -.31 \\
\hline âge & .01 & .21 & .05 & .04 \\
\hline expérience & .17 & .23 & .08 & .07 \\
\hline $\begin{array}{l}\text { quotidiens grand } \\
\text { format de Montréal } \\
\text { et de Québec }\end{array}$ & $.22^{*}$ & .29 & $-.31^{*}$ & -.32 \\
\hline poste & .05 & .07 & -.08 & -.12 \\
\hline revenus & - & .02 & .10 & .15 \\
\hline syndicalisation & .05 & .04 & -.01 & -.03 \\
\hline $\mathrm{R}^{2}$ et $\mathrm{R}$ & .12 & .34 & .21 & .45 \\
\hline constante & \multirow{2}{*}{\multicolumn{2}{|c|}{$\begin{array}{r}3.4 \\
130 .\end{array}$}} & \multirow{2}{*}{\multicolumn{2}{|c|}{$\begin{array}{r}8.5 \\
130 .\end{array}$}} \\
\hline $\mathrm{N}$ & & & & \\
\hline
\end{tabular}

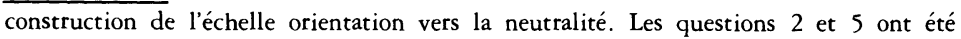
rejetées afin d'assurer une meilleure consistance interne des échelles. 
C'est le type d'entreprise dans laquelle travaillent les journalistes qui permet le mieux de prédire la position occupée sur chacune des échelles. Les journalistes à l'emploi des quotidiens d'information grand format de Québec et de Montréal sont plus orientés vers la participation (beta: .22) et ils ont moins tendance à privilégier la neutralité (beta: - - 31) que les journalistes travaillant dans les journaux du groupe Québécor ou dans les quotidiens régionaux (tableau 7). Ce résultat va dans le sens du diagnostic esquissé plus haut: la nature des entreprises de presse elles-mêmes semble déterminante dans la définition de l'attitude professionnelle des journalistes. Nos résultats sont similaires à ceux qu'ont rapportés Johnstone et alii dans leur enquête menée aux États-Unis. Ceux-ci ont montré que les journalistes américains qui privilégiaient l'analyse et l'enquête se retrouvaient surtout dans les grandes villes et dans la presse la plus prestigieuse. ${ }^{13}$

Le poste occupé dans le journal permet aussi de prédire le rang obtenu sur chacune des deux échelles. Pour les fins de l'analyse, nous avons séparé ici les journalistes en deux groupes: le premier groupe comprend les généralistes et les journalistes affectés aux sports, y compris les préposés au pupître, et le second, les chroniqueurs spécialisés et les journalistes occupant des fonctions de direction, syndiquées ou non. Il apparaît que les seconds ont tendance à privilégier davantage la participation et qu'ils sont moins orientés vers la neutralité que les généralistes et les rédacteurs sportifs.

Certaines caractéristiques personnelles contribuent aussi à structurer l'orientation professionnelle des journalistes. C'est notamment le cas pour le niveau de scolarité. Il apparaît clai-

13. La comparaison avec le travail de Johnstone et alii doit ici être considérée à titre indicatif pour deux raisons. Les deux échelles des chercheurs américains comprennent quelques indicateurs supplémentaires que nous n'avons pas recueillis lors de notre propre enquête. De plus, le nombre de variables incluses dans leur modèle est plus élevé que dans celui que nous proposons. 
rement qu'une position élevée sur l'échelle de participation est associée à un niveau de scolarité élevé (beta: .07), alors qu'au contraire, les journalistes qui ont un niveau de scolarité moins élevé ont tendance à favoriser la neutralité (beta: -.23 ). Ces résultats vont tout à fait dans le sens de ce qu'ont observé Johnstone et ses collaborateurs, qui ont eux aussi noté la relation en sens opposé entre le niveau de scolarité et les deux échelles. Cette constatation donne à penser que la tendance à la neutralité, surtout partagée par les répondants moins scolarisés qui ont fait leur apprentissage du métier de journaliste sur le tas, serait acquise au cours de la pratique quotidienne, alors que les personnes plus scolarisées auraient tendance à aborder la pratique de ce métier avec une orientation théorique déjà articulée, tendant vers un journalisme plus intervenant. ${ }^{14}$

Une fois contrôlées les autres variables, en particulier la scolarité, l'âge des répondants paraît faiblement associé à la conception qu'ils se font du métier de journaliste. Ce résultat est important car l'examen de la relation entre l'âge et l'échelle de participation laisse entendre que les jeunes journalistes seraient moins participants $(\mathrm{R}: .21)$. Or, il n'en est rien, car le coefficient décrivant l'effet de l'âge tombe à .01 dans l'analyse de régression, ce qui indique que cette variable est négligeable dans la prédiction de l'orientation vers la participation. Le nombre d'années d'expérience est ici plus important que l'âge pour annoncer l'orientation professionnelle, surtout dans le cas de l'échelle de participation. Cette dernière observation va à l'encontre de celle de Johnstone et alii, qui avaient observé que l'âge avait un poids plus grand que l'expérience.

Cette analyse apporte une réponse claire à la question déjà posée au terme de l'examen de la typologie et elle confirme que les entreprises de presse jouent un rôle clé dans l'orientation

14. Johnstone, J.W.C. et alii, The News People, Urbana, Ill., University of Illinois Press, 1976, 257 p. 
du journalisme au Québec. Non seulement contribuent-elles à structurer le profil de ceux qui entrent dans ce type de carrière, mais encore, l'appartenance à un type donné d'entreprise et le milieu de travail qu'on y retrouve influencent-ils grandement la conception que les journalistes se font de leur métier.

\section{c) Profil du bon journaliste}

Une autre façon de caractériser la représentation sociale de leur métier consiste à mesurer les opinions des répondants vis-àvis d'un certain nombre de stéréotypes ou d'images. Nous leur avons demandé de dégager à partir d'une liste comprenant 10 items les trois qualités qui apparaissent le mieux définir un bon journaliste (tableau 8). L'honnêteté est de loin la qualité la plus souvent mentionnée (73.8\%). Viennent ensuite «les contacts personnels et l'expérience», choisie par $44.3 \%$ des répondants (avec un sommet de $63.3 \%$ dans le cas des journalistes à l'emploi de Québécor), et l'objectivité à 40.9\% (60\% chez

\section{TABLEAU 8}

Qualité du bon journaliste selon le type de journal

\begin{tabular}{lccccc}
\hline & $\begin{array}{c}\text { Quotidiens } \\
\text { grand format } \\
\text { Quntréal et Québec }\end{array}$ & $\begin{array}{c}\text { Quotidiens } \\
\text { Québécor }\end{array}$ & $\begin{array}{c}\text { Quotidiens } \\
\text { régionaux }\end{array}$ & Total & $\mathrm{p}$ \\
\cline { 1 - 4 } objectivité & 36. & 60. & 36.7 & 40.9 & .13 \\
contacts & 39.5 & 63.3 & 40. & 44.3 & .13 \\
indépendance & 48.8 & 20. & 33.3 & 39.6 & .04 \\
honnêteté & 72.1 & 80. & 73.3 & 73.8 & n.s. \\
prudence & 5.8 & 3.3 & 16.7 & 8.7 & $<.001$ \\
imagination & 32.6 & 10. & 36.7 & 28.9 & .08 \\
minutie & 26.7 & 13.3 & 13.3 & 20.8 & .19 \\
bonne perception & 18.6 & 26.7 & 26.7 & 22.8 & n.s. \\
audace & 18.6 & 20. & 23.3 & 19.5 & n.s. \\
\hline
\end{tabular}


Québécor). Il n'y a rien d'étonnant à ce que les répondants valorisent beaucoup plus l'honnêteté que l'objectivité. À notre avis, en effet, bon nombre de journalistes québécois ont la conviction que le journaliste ne peut toujours présenter que sa propre perception des événements qu'il est appelé à couvrir, que ses choix sont toujours influencés par son milieu, ses valeurs, voire ses préjugés dont, par souci d'honnêteté, il doit toutefois chercher à se détacher (voir le film de J. Godbout, Feu l'objectivité, Office national du film, 1979). Aux États-Unis, où les discussions sur l'objectivité se sont accentuées au cours des années soixante en même temps que le mouvement pour un journalisme participant gagnait en importance, certains ont parlé de "mature subjectivity" ${ }^{15}$. Mais ce débat n'a rien de neuf, puisque dès les années vingt, le fondateur du magazine Time, Henry Luce, disait: "Show me a man who says he is objective and I'll show you a man who is deceiving himself » (cité par Schudson).

De toutes les qualités suggérées, les répondants retiennent, presque tout autant que l'objectivité, l'indépendance d'esprit dont on pourrait dire qu'elle se rattache plutôt à la presse d'opinion et fait contrepoids, d'une certaine façon, à l'objectivité, née avec la presse d'information à laquelle elle était éssentielle. Comment espérer en effet vendre son journal aux uns et aux autres, à la droite et à la gauche, aux conservateurs et aux libéraux, sans rester le plus impartial ou le plus objectif possible? C'est ainsi que l'indépendance d'esprit retient davantage l'attention des éducateurs et des enquêteurs-analystes, alors que, chez les séducteurs, on ne veut pas en entendre parler. Les journalistes, s'ils accordent peu d'importance à l'audace, ne s'embarrassent guère non plus de la prudence, à l'exception des

15. Schudson, Michael, Discovering the news, New York, Basic books, 1978, 228 p. 
chroniqueurs spécialisés (sauf les sportifs) et des journalistes de la presse régionale, plus prudents peut-être à cause des liens plus fréquents et plus immédiats qu'ils doivent entretenir avec leurs sources d'information. Notons aussi que les journalistes qui occupent des postes de direction accordent plus de place à l'imagination et enfin, que les séducteurs, conscients du marché, sont plus nombreux à juger nécessaire d'avoir une bonne perception du public.

\section{La pratique du métier}

Nous ne pouvons pas nous limiter à caractériser la pratique du journalisme au Québec à partir de la seule représentation que se font les journalistes de leur rôle. Il faut aussi voir jusqu'à quel point leur pratique effective et quotidienne correspond à l'image qu'ils se donnent de leur métier. Nous aborderons cette question en dégageant l'évaluation que font les journalistes de la possibilité d'exercer leur métier dans l'entreprise qui les emploie. Comme il s'agit là d'une évaluation globale, nous chercherons à cerner de façon plus précise leur travail en distinguant quelques points de repère qui guident les journalistes dans la rédaction de leurs textes. Sont-ils sensibles aux critiques de leurs collègues ou de leurs pairs? Écrivent-ils en fonction des besoins de mise en marché de leur entreprise? Doivent-ils tenir compte des pressions des sources d'information?

\section{a) L'exercice effectif du métier}

En très forte majorité, les journalistes qui se définissent comme reporters ou comme séducteurs disent qu'ils n'ont pas de difficultés à exercer leur rôle (tableau 9). Il en va autrement pour les enquêteurs-analystes et pour ceux qui se définissent comme éducateurs puisqu'ils soutiennent en plus fortes pro- 
TABLEAU 9

Typologie des journalistes de la presse quotidienne selon la perception qu'ils ont de la possibilité de jouer leur rôle dans le journal qui les emploie

\begin{tabular}{lccccc}
\hline $\begin{array}{l}\text { Types de } \\
\text { journalistes }\end{array}$ & entièrement & assez & un peu & pas du tout & total \\
& & & & & peuvent jouer leur rôle \\
& 12.5 & 34.4 & 50. & 3.1 & 100. \\
\hline $\begin{array}{l}\text { éducateur } \\
\text { enquêteur- }\end{array}$ & 26.1 & 39.1 & 30.4 & 4.4 & 100. \\
$\begin{array}{l}\text { analyste } \\
\text { reporter }\end{array}$ & 48.9 & 42.6 & 6.4 & 2.1 & 100. \\
séducteur & 60.9 & 34.8 & 4.3 & - & 100. \\
\hline Total & 35.8 & 38.5 & 23. & 2.7 & 100. \\
\hline
\end{tabular}

$\mathrm{p}<.001$

portions qu'ils ne peuvent pas exercer pleinement le rôle qu'ils valorisent dans le journal qui les emploie. Des différences notables se retrouvent aussi lorsqu'on examine le poste occupé dans le journal. Les généralistes soutiennent plus fréquemment que les autres catégories de journalistes qu'ils ne peuvent exercer le rôle qu'ils jugent le plus important, suivi des journalistes qui détiennent des fonctions de direction, les plus satisfaits étant par contre les journalistes sportifs. Ici encore, les journalistes du groupe Québécor se distinguent des autres puisqu'ils mentionnent en majorité ne pas avoir de problèmes, entre autres parce que les reporters et les séducteurs y sont plus nombreux.

Les journalistes qui ont une conception de leur métier que l'on pourrait qualifier de traditionnelle (rapporter la nouvelle) ou de commerciale (intéresser le lecteur) peuvent donc exercer leur métier comme ils l'entendent dans la presse écrite québécoise. Ceux qui voudraient au contraire développer l'analyse et 
ceux qui tentent de décortiquer ce que les agents d'information et les relationnistes à l'emploi des divers appareils cherchent à livrer au public par le biais du journal, ne trouvent pas toujours la possibilité d'exercer cette fonction efficacement dans le quotidien qui les emploie. C'est ce qui ressort nettement lorsqu'on met en relation l'évaluation que font les journalistes de la possibilité d'exercer leur métier et l'échelle de participation que nous avons déjà présentée plus haut: les tenants d'un journalisme participant sont les plus critiques quand on leur demande s'ils peuvent jouer leur rôle selon leur désir. Ces résultats permettent de mieux comprendre les conflits relatifs à la gestion des salles de rédaction et aux contenus d'information (les clauses professionnelles) qui ont secoué La Presse, Le Soleil et Le Devoir ces dernières années. Nous retrouvons dans ces trois quotidiens le plus grand nombre de partisans d'un journalisme plus actif et ce sont eux qui font part en plus fortes proportions d'un fossé entre leur conception du métier et la possibilité de le pratiquer conformément à cette dernière.

Par contre, les longues grèves des dernières années au sujet des clauses professionnelles pourraient bien avoir usé ces journalistes qui, s'ils ont conservé leurs idéaux, ont maintenant d'autres soucis plus immédiats. Lorsqu'on leur demande de marquer leur choix entre deux types de gains devant faire l'objet d'une négociation collective en 1981: les gains matériels et l'accroissement des garanties de nature professionnelle, la majorité des journalistes qui sont à l'emploi des journaux métropolitains grand format, qui ont aussi vieilli et qui ont sans doute contracté des obligations financières importantes propres à leur groupe d'âges, mettent maintenant l'accent sur les gains d'ordre matériel. Au contraire, une très forte majorité des journalistes appartenant au groupe Québécor (83.3\%) ou travaillant dans les journaux régionaux mentionnent les garanties professionnelles (tableau 10). Notons cependant que le concept de garanties 
TABLEAU 10

Type de revendications devant faire l'objet de négociations collectives selon le type d'entreprises de presse

\begin{tabular}{lcccc}
\hline Revendications & $\begin{array}{c}\text { Quotidiens } \\
\text { grand format } \\
\text { Montréal et Québec }\end{array}$ & $\begin{array}{c}\text { Quotidiens } \\
\text { Québécor }\end{array}$ & $\begin{array}{c}\text { Quotidiens } \\
\text { régionaux }\end{array}$ & Total \\
\hline $\begin{array}{l}\text { Gains matériels } \\
\begin{array}{l}\text { Garanties } \\
\text { professionnelles }\end{array}\end{array}$ & 59.0 & 16.7 & 25.0 & 43.6 \\
\hline & 41.0 & 83.3 & 75.0 & 56.4 \\
\hline TOTAL & 100.0 & 100.0 & 100.0 & 100.0 \\
\hline $\mathrm{p}<.001$ & 83 & 30 & 28 & 141 \\
\hline
\end{tabular}

professionnelles semble avoir évolué depuis quelques années. Dans l'esprit des journalistes de Québécor, il ne s'agit pas de revendiquer plus de pouvoir en matière d'information, comme l'ont fait leurs collègues des quotidiens grand format de Montréal et de Québec. Ainsi, des journalistes du Journal de Montréal ont clairement exprimé lors d'interviews qu'ils ne désiraient en rien changer la vocation du journal dont ils semblent partager en gros les objectifs; on veut simplement plus d'espace pour les nouvelles et un minimum de structures dans la salle de rédaction ${ }^{16}$. Nos résultats viennent tout de même appuyer de façon éloquente un diagnostic que posait Lysiane Gagnon en 1980: «En réalité, si l'on fait exception d'une partie de la presse régionale, de la radio privée et de l'empire Québécor, où toutes les grandes batailles syndicalo-professionnelles restent à faire, force est de constater que partout où les syndicats sont

16. Sauvageau, Florian, «La presse francophone et ses artisans», dans Commission Royale sur les quotidiens, Du côté des Journalistes, Publications de recherches, volume 2, Ottawa, 1981, 43-52. 
TABLEAU 11

Type de revendications devant faire l'objet de négociations collectives selon le poste occupé par le journaliste dans l'entreprise

\begin{tabular}{lccccc}
\hline Revendications & Direction & $\begin{array}{c}\text { Chroniqueur } \\
\text { spécialisé }\end{array}$ & Sport & Généraliste & Total \\
\hline $\begin{array}{l}\text { Gains matériels } \\
\begin{array}{l}\text { Garanties } \\
\text { professionnelles }\end{array}\end{array}$ & 23.8 & 37.3 & 47.8 & 54.3 & 43.6 \\
\hline & 16.2 & 62.7 & 52.2 & 45.7 & 56.4 \\
TOTAL & 100.0 & 100.0 & 100.0 & 100.0 & 100.0 \\
& 21 & 51 & 23 & 46 & 141 \\
\hline
\end{tabular}

$\mathrm{p}=.09$

solidement implantés, le temps des luttes de principe tire à sa fin... ${ }^{17}$

Nos données permettent aussi de voir que la nature des revendications est différente selon la fonction occupée dans le journal (tableau 11). Les journalistes qui sont rattachés à la direction, y compris les éditorialistes, et les chroniqueurs spécialisés penchent davantage vers la recherche de gains d'ordre professionnel alors que les généralistes et les journalistes sportifs recherchent plutôt des gains matériels à travers les négociations collectives. Par ailleurs, la conception que les journalistes de la presse quotidienne se font de leur métier affecte peu le sens de leurs revendications: leur répartition entre quatre groupes différents - l'éducateur, l'enquêteur, le reporter et le séducteur - fait beaucoup moins varier le choix de ce qui devrait être l'objet de négociations collectives.

17. Gagnon, Lysiane, «Journaliste et syndiqué: le perpétuel dilemme», dans F. Sauvageau, G. Lesage et J. De Bonville (eds), Les Journalistes, Montréal, QuébecAmérique, 1980: 43-72. 
TABLEAU 12

Facteurs dont les journalistes doivent tenir compte dans l'exercice de leur métier, selon le type d'entreprise de presse

\begin{tabular}{|c|c|c|c|c|c|c|}
\hline Facteurs & & $\begin{array}{c}\text { Quotidiens } \\
\text { grand format } \\
\text { Montréal et Québec }\end{array}$ & $\begin{array}{l}\text { Quotidiens } \\
\text { Québécor }\end{array}$ & $\begin{array}{l}\text { Quotidiens } \\
\text { régionaux }\end{array}$ & Total & \\
\hline $\begin{array}{l}\text { Besoins de } \\
\text { marketing } \\
\text { de l'entreprise }\end{array}$ & $\begin{array}{c}\text { oui } \\
\text { en partie } \\
\text { non }\end{array}$ & $\begin{array}{r}8.1 \\
46.6 \\
45.3\end{array}$ & $\begin{array}{l}26.7 \\
46.6 \\
26.7\end{array}$ & $\begin{array}{r}3.6 \\
57.1 \\
39.3\end{array}$ & $\begin{array}{l}11.1 \\
48.6 \\
40.3\end{array}$ & $p=.02$ \\
\hline $\begin{array}{l}\text { Réactions } \\
\text { des collegues }\end{array}$ & $\begin{array}{c}\text { oui } \\
\text { en partie } \\
\text { non }\end{array}$ & $\begin{array}{r}2.4 \\
30.5 \\
67.1\end{array}$ & $\begin{array}{r}6.9 \\
24.1 \\
69.0\end{array}$ & $\begin{array}{r}7.4 \\
23.3 \\
59.3\end{array}$ & $\begin{array}{r}4.3 \\
29.7 \\
66.0\end{array}$ & $p=$ n.s. \\
\hline $\begin{array}{l}\text { Pressions } \\
\text { des sources } \\
\text { d'information }\end{array}$ & $\begin{array}{c}\text { oui } \\
\text { en partie } \\
\text { non }\end{array}$ & $\begin{array}{r}1.2 \\
40.7 \\
58.1\end{array}$ & $\begin{array}{l}10.3 \\
41.4 \\
48.3\end{array}$ & $\begin{array}{l}14.3 \\
57.1 \\
28.6\end{array}$ & $\begin{array}{r}5.6 \\
44.1 \\
50.3\end{array}$ & $p=.01$ \\
\hline $\begin{array}{l}\text { Attentes } \\
\text { du public }\end{array}$ & $\begin{array}{l}\text { oui } \\
\text { en partie } \\
\text { non }\end{array}$ & $\begin{array}{r}74.4 \\
24.4 \\
1.2\end{array}$ & $\begin{array}{r}93.2 \\
3.4 \\
3.4\end{array}$ & $\begin{array}{l}76.7 \\
23.3 \\
-\end{array}$ & $\begin{array}{r}78.6 \\
20.0 \\
1.4\end{array}$ & $p=.12$ \\
\hline
\end{tabular}




\section{b) Quelques points de repère dans l'exercice du métier}

Les journalistes sont aussi engagés dans divers systèmes d'interaction qui exercent des contraintes sur leurs façons de travailler. Le premier de ces systèmes est formé par les pairs, dans la salle de rédaction. À cet égard les journalistes se disent très peu préoccupés des réactions de leurs collègues de travail, quels que soient le type d'entreprise de presse, la fonction occupée dans le journal ou leurs orientations personnelles (tableau 12). Ce résultat ne signifie pas que les journalistes travaillent sans se référer à ce qu'écrivent leurs collègues et n'infirme pas d'autres travaux, comme ceux de Jean de Bonville qui avait constaté que les journalistes étaient de grands consommateurs d'information en notant que «la lecture des autres journaux servait à l'acquisition de modèles et de méthodes de travail ${ }^{\mathbf{1 8}}$. Les journalistes peuvent en effet s'inspirer de leurs collègues des autres médias affectés à la couverture des mêmes secteurs d'activités qu'eux sans se soucier de leurs collègues de travail attachés à d'autres rubriques. Ainsi, le chroniqueur syndical d'un journal pourra bien ne pas se préoccuper de la réaction de ses collègues chroniqueurs politiques tout en accordant de l'importance à ce qu'écrivent les chroniqueurs syndicaux des journaux concurrents. Par ailleurs, nos résultats confirment le grand souci des journalistes de veiller à l'intégrité de leurs textes, que certains vont même jusqu'à considérer comme une sorte d'acte journalistique, souci qui les a amenés à négocier des clauses professionnelles rigides qui empêchent le pupitre (les préposés à la vérification) de modifier le contenu d'un article sans l'autorisation de son auteur, à moins d'éliminer la signature.

Si les journalistes disent peu s'inquiéter des réactions de leurs collègues de travail, il en va autrement pour ce qui est des

18. De Bonville, Jean, Le journaliste et sa documentation: sources d'information et habitudes documentaires des journalistes de la presse francophone du Québec, Québec, EdiGric, Université Laval, 1977, 236 p. 
besoins de marketing des journaux. Au total, $60 \%$ des journalistes confient en tenir compte, au moins en partie, dans l'exercice de leur métier. Nous avions retenu cet indicateur parce que les journaux, sauf des exceptions comme Le Devoir, sont des entreprises commerciales soumises à l'impératif du profit et que les soucis de marketing y ont pris une importance accrue ces dernières années. Si la fonction du journaliste est au fond de fabriquer de l'information, celle de l'entreprise consiste à la vendre à un public donné qu'elle doit intéresser, en l'arrachant en quelque sorte aux autres médias et à une multitude d'activités de loisirs, toutes en concurrence avec la presse quotidienne. En fait, si les journaux avaient échappé, grâce à la publicité, à la tutelle de l'État et des partis qui asservissait la presse d'opinion, c'était pour trouver une autre servitude, celle du profit, qui les oblige à concevoir le produit qui réunira le plus de lecteurs possible, qu'on pourra vendre aux annonceurs à meilleur prix s'ils sont plus nombreux.

En 1977, les journalistes du Soleil faisaient la grève pour empêcher la «commercialisation» de leur journal qu'on voulait détourner, disaient-ils, de sa mission d'information. Ils se battaient contre l'information-spectacle, contre ce qu'on appelle maintenant le disco-journalisme. Mais la disparition de quelques journaux (Montréal-Matin, The Montreal Star), la baisse ou au mieux la stagnation du tirage de certains autres, la diminution des revenus publicitaires dans le contexte de crise économique, inquiètent maintenant les journalistes qui savent que les journaux sont vulnérables. Sans doute acceptent-ils mieux aujourd'hui qu'il y a dix ans que leur journal se préoccupe de marketing. Les journalistes appartenant au groupe Québécor paraissent cependant plus favorables à cette dimension que leurs collègues ouvrant dans la presse régionale ou dans les journaux grand format de Québec et de Montréal. De même, les séducteurs et les reporters sont beaucoup plus sensibles aux 
impératifs de marketing que leurs collègues éducateurs ou enquêteurs-analystes.

Enfin, les journalistes se disent dans l'ensemble peu soumis aux pressions des sources d'information, sauf dans le cas de la presse régionale. Dans les villes de taille moyenne en effet, les journalistes doivent compter avec les pressions inhérentes à un milieu plus restreint, d'autant plus que les quotidiens régionaux se perçoivent souvent comme des outils au service de la réalisation de projets locaux ${ }^{19}$. C'est dans le même esprit qu'il faut comprendre leur plus grande prudence, comme nous l'avons déjà souligné. Par contre, il ne faut pas se surprendre que les journalistes qui fréquentent plutôt des pouvoirs "nationaux» n'aient pas à se soucier des pressions ouvertes de leurs sources d'information; la cohorte des conseillers en communication qui entoure ces divers pouvoirs (politiques, syndicaux, économiques) connait des façons plus subtiles d'influencer la presse et qui paraissent moins évidentes.

\section{IV Évaluation du journalisme pratiqué au Québec}

On retrouve le même clivage entre les types de quotidiens et de journalistes lorsque les répondants évaluent la qualité du journalisme pratiqué au Québec. $90 \%$ des répondants à l'emploi de Québécor ou des quotidiens régionaux considèrent le journalisme québécois comme «bon» ou «très bon». Les reporters et les séducteurs l'évaluent de la même façon, dans des proportions à peu près identiques. Au contraire, plus du tiers des éducateurs et plus de $30 \%$ des enquêteurs-analystes ont une attitude plutôt négative vis-à-vis du travail de leurs collègues et estiment le journalisme québécois «passable» ou «de mau-

19. Tremblay, B., "Grandeurs et misères de la presse régionale», dans Sauvageau F., G. Lesage et J. De Bonville, Les Journalistes, Montréal, QuébecAmérique, 1980: 203-238. 
vaise qualité ». Pas un seul des journalistes que nous avons définis comme enquêteurs-analystes ne juge «très bon» le jourlisme d'ici, l'esprit critique étant particulièrement développé au Devoir, à La Presse et au Soleil.

L'analyse des reproches précis que les journalistes interrogés font à la presse permet d'éclairer ce jugement global qu'ils portent sur les quotidiens québécois (tableau 13). Ils pensent en majorité que les articles ne sont pas assez bien documentés et que souvent les journaux ne s'intéressent qu'au spectaculaire. Ces critiques sont évidemment plus prononcées dans le groupe des journalistes éducateurs et analystes, dont l'évaluation générale est plus sévère. Fait à noter, peu de journalistes font grief à leurs collègues de manquer d'objectivité, contrairement à un reproche qui est couramment véhiculé dans l'opinion publique à propos de la presse.

Une autre critique est maintes fois adressée à la presse, soit celle de servir de haut-parleur aux pouvoirs. Ainsi, quelques

TABLEAU 13

Opinions des journalistes sur la presse écrite

\begin{tabular}{lcccccc}
\hline & $\begin{array}{c}\text { Entièrement } \\
\text { d'accord }\end{array}$ & $\begin{array}{c}\text { Assez en } \\
\text { accord }\end{array}$ & $\begin{array}{c}\text { Peu } \\
\text { d'accord }\end{array}$ & $\begin{array}{c}\text { Pas du tout } \\
\text { d'accord }\end{array}$ & $\begin{array}{c}\text { Total } \\
\%\end{array}$ & $\mathrm{~N}$ \\
\hline $\begin{array}{l}\text { les articles des jour- } \\
\text { naux ne sont pas assez } \\
\text { documentés }\end{array}$ & 14.8 & 58.4 & 22.8 & 4. & 100 & 149 \\
$-\begin{array}{l}\text { les journaux ne s'inté- } \\
\text { ressent qu'au specta- }\end{array}$ & 14.8 & 49.6 & 30.2 & 5.4 & 100 & 149 \\
$\begin{array}{l}\text { culaire } \\
- \text { les articles sont trop }\end{array}$ & 16.8 & 35.6 & 36.9 & 10.7 & 100 & 149 \\
$\begin{array}{l}\text { longs } \\
- \text { les journalistes man- } \\
\text { quent d'objectivité }\end{array}$ & 4.7 & 12.8 & 57.5 & 25. & 100 & 149 \\
$-\begin{array}{l}\text { les journaux sont trop } \\
\text { intellectuels }\end{array}$ & 14.1 & 20.8 & 43.6 & 21.5 & 100 & 149 \\
$-\begin{array}{l}\text { les journalistes ont } \\
\text { peur de s'engager }\end{array}$ & 14.8 & 24.8 & 40.9 & 19.5 & 100 & 149 \\
\hline
\end{tabular}


études ont été consacrées, dans le cadre des travaux de l'Institut canadien d'éducation des adultes, à la place privilégiée qu'accorderaient les médias au pouvoir politique par exemple, au détriment des syndicats et des groupes «populaires » et "progressistes». Qu'on pense à l'analyse de la couverture du conflit qui a paralysé les services de transport en commun à Montréal en janvier 1982, dont l'I.C.E.A. a déduit que les médias avaient «favorisé l'information gouvernementale et patronale et minimisé le point de vue syndical», ou aux recherches sur les actualités télévisées publiées en 1979, faites avec avec la collaboration de chercheurs de l'UQAM, et selon lesquelles dans $67 \%$ des cas «les déclencheurs de la nouvelle sont des opinions et des réflexions d'hommes politiques».

Nous aborderons cette question par un biais moins bien connu: celui de la perception qu'ont les journalistes du traitement accordé par la presse aux différents acteurs sociaux. Dans l'ensemble, les journalistes de la presse écrite quotidienne s'entendent pour affirmer que les hommes politiques et les sportifs reçoivent une très large couverture dans les quotidiens. Les opinions des gens d'affaires et des chefs syndicaux leur semblent moins bien véhiculées, tandis que celles qui viennent des groupes populaires ou des femmes en général leur paraissent occuper une place encore moins importante. La perception des journalistes varie assez peu à cet égard selon le type de presse et le poste qu'ils occupent dans l'entreprise. Par contre, ceux qui se définissent comme éducateurs et enquêteurs-analystes sont plus nombreux à estimer que les opinions des groupes populaires sont sous-représentées (tableau 14). Un avis que les rédacteurs sportifs, comme les séducteurs, ne partagent absolument pas.

On peut dire que, d'une certaine façon, et même si leur vision est moins manichéenne que celle de l'I.C.E.A. (le pouvoir politique et patronal sur-valorisé vs des syndicats et groupes 
TABLEAU 14

«Les opinions des groupes suivants sont-elles bien véhiculées par les quotidiens?», selon la typologie des journalistes

\begin{tabular}{|c|c|c|c|c|c|c|}
\hline & & Éducateur & Analyste & Reporter & Séducteur & \\
\hline $\begin{array}{l}\text { Hommes } \\
\text { politiques }\end{array}$ & $\begin{array}{l}\text { Très bien } \\
\text { Assez bien } \\
\text { Peu } \\
\text { Pas du tout }\end{array}$ & $\begin{array}{c}93.5 \\
6.5 \\
- \\
-\end{array}$ & $\begin{array}{l}88.9 \\
11.1 \\
- \\
-\end{array}$ & $\begin{array}{r}91.5 \\
6.4 \\
2.1 \\
-\end{array}$ & $\begin{array}{l}78.3 \\
21.7 \\
- \\
-\end{array}$ & $\mathrm{p}=\mathrm{n} . \mathrm{s}$. \\
\hline $\begin{array}{l}\text { Gens } \\
\text { d'affaires }\end{array}$ & $\begin{array}{l}\text { Très bien } \\
\text { Assez bien } \\
\text { Peu } \\
\text { Pas du tout }\end{array}$ & $\begin{array}{l}43.8 \\
43.8 \\
12.4 \\
-\end{array}$ & $\begin{array}{c}32.6 \\
62.0 \\
4.4 \\
-\end{array}$ & $\begin{array}{c}43.5 \\
54.3 \\
2.2 \\
-\end{array}$ & $\begin{array}{l}34.8 \\
47.8 \\
17.4 \\
-\end{array}$ & $\mathrm{p}=.17$ \\
\hline $\begin{array}{l}\text { Chefs } \\
\text { syndicaux }\end{array}$ & $\begin{array}{l}\text { Très bien } \\
\text { Assez bien } \\
\text { Peu } \\
\text { Pas du tout }\end{array}$ & $\begin{array}{r}56.3 \\
37.5 \\
6.3 \\
-\end{array}$ & $\begin{array}{c}47.8 \\
43.5 \\
8.7 \\
-\end{array}$ & $\begin{array}{l}66.0 \\
34.0 \\
- \\
-\end{array}$ & $\begin{array}{c}43.5 \\
52.2 \\
4.3 \\
-\end{array}$ & $\mathrm{p}=\mathrm{n} . \mathrm{s}$. \\
\hline Les sportifs & $\begin{array}{l}\text { Très bien } \\
\text { Assez bien } \\
\text { Peu } \\
\text { Pas du tout }\end{array}$ & $\begin{array}{c}90.6 \\
9.4 \\
- \\
-\end{array}$ & $\begin{array}{l}84.1 \\
15.9 \\
- \\
-\end{array}$ & $\begin{array}{c}91.3 \\
8.7 \\
- \\
-\end{array}$ & $\begin{array}{r}91.4 \\
4.3 \\
4.3 \\
-\end{array}$ & $\mathrm{p}=\mathrm{n} . \mathrm{s}$. \\
\hline $\begin{array}{l}\text { Groupes } \\
\text { populaires }\end{array}$ & $\begin{array}{l}\text { Très bien } \\
\text { Assez bien } \\
\text { Peu } \\
\text { Pas du tout }\end{array}$ & $\begin{array}{r}9.7 \\
38.7 \\
48.4 \\
3.2\end{array}$ & $\begin{array}{r}17.4 \\
30.4 \\
47.8 \\
4.3\end{array}$ & $\begin{array}{l}14.9 \\
59.6 \\
25.5 \\
-\end{array}$ & $\begin{array}{l}26.1 \\
56.5 \\
17.4 \\
-\end{array}$ & $\mathrm{p}=.04$ \\
\hline Les femmes & $\begin{array}{l}\text { Très bien } \\
\text { Assez bien } \\
\text { Peu } \\
\text { Pas du tout }\end{array}$ & $\begin{array}{l}15.6 \\
46.9 \\
37.5 \\
-\end{array}$ & $\begin{array}{l}19.6 \\
32.6 \\
47.8 \\
-\end{array}$ & $\begin{array}{l}15.2 \\
52.2 \\
32.6 \\
-\end{array}$ & $\begin{array}{l}26.1 \\
43.5 \\
30.4 \\
-\end{array}$ & $\mathrm{p}=\mathrm{n} . \mathrm{s}$. \\
\hline TOTAL & $\begin{array}{l}\% \\
\mathrm{~N}\end{array}$ & $\begin{array}{l}100.0 \\
32\end{array}$ & $\begin{array}{c}100.0 \\
46\end{array}$ & $\begin{array}{l}100.0 \\
46\end{array}$ & $\begin{array}{c}100.0 \\
23\end{array}$ & \\
\hline
\end{tabular}

populaires sous-représentés), les journalistes reconnaissent en général que les acteurs les moins bien organisés trouvent plus difficilement leur place dans les quotidiens. Ils s'entendent tous par ailleurs pour souligner que les sportifs et les hommes poli- 
tiques y trouvent sans problème un véhicule pour leurs opinions. Il y a en fait une certaine parenté entre ces deux groupes: ils s'offrent en spectacle. Et il n'y a rien d'étonnant à ce que la presse, qui préfere les personnalités aux idées, supposément parce qu'elles se vendent mieux, accorde un traitement privilégié à ceux des acteurs sociaux dont la popularité ou la notoriété risque le plus, croit-on, de retenir l'attention d'un vaste public (d'autant plus qu'ils mettent tout en œuvre pour qu'on parle d'eux). Cela tend à renforcer la thèse de ceux qui, comme l'I.C.E.A., ont accordé tant d'importance ces dernières années à l'influence à long terme du mode de propriété et des patrons sur les contenus véhiculés par les médias. Malgré les garanties importantes d'indépendance professionnelle négociées par les syndicats de journalistes depuis une quinzaine d'années, les priorités des entreprises de presse, les budgets affectés aux divers secteurs d'activités, restent en effet définis par la seule direction ou par les cadres qu'elle a choisis.

\section{Conclusion}

Une même observation revient souvent dans l'analyse des données qui vient d'être proposée: celle du poids important de l'entreprise de presse dans la définition des orientations du journalisme écrit au Québec. Nous avons montré que ce sont les entreprises qui déterminent le profil moyen des journalistes en définissant les conditions d'embauche, fort variables d'un journal à l'autre. De même la conception de la pratique du journalisme est apparue fortement dépendante de la nature de l'entreprise de presse. L'entreprise, ou l'employeur, sert aussi à distinguer les journalistes satisfaits et les mécontents; les journalistes du groupe Québécor (des séducteurs et des reporters pour la plupart) considèrent en grande majorité pouvoir jouer pleinement leur rôle dans le cadre de leur emploi; les enquêteurs-analystes et les éducateurs au contraire, tenants d'un journalisme plus 
actif et plus nombreux dans les quotidiens grand format de Québec et de Montréal ont du mal à exercer leur métier tel qu'ils l'entendent.

Il est intéressant de noter que c'est dans les entreprises (La Presse, Le Soleil) qui, manifestement, se sont cherché une voie ces dernières années, à la fois secouées et tentées par le succès de la formule des quotidiens Québécor, que les journalistes font part des plus grandes frustrations (au Devoir, le mécontentement peut être attribuable au fait que le sondage ait été effectué peu de temps avant la grève qui a paralysé le journal au printemps 1981). Les journalistes à l'emploi de ces quotidiens-omnibus partagent pourtant les inquiétudes que leurs patrons ont eues (s'ils ne les ont pas encore) face au futur. Les enquêteurs-analystes et les éducateurs qu'on retrouve en plus grand nombre dans les quotidiens grand format de Montréal et de Québec sont en effet les plus pessimistes au sujet de l'avenir de la presse quotidienne, dont ils sont aussi les critiques les plus sévères. Sans doute parce qu'ils considèrent que l'avenir n'est pas, pour leur journal, dans l'imitation des dernières formules à la mode, que d'autres ont déjà appris depuis longtemps à mieux maîtriser, mais du côté d'un journalisme original et personnel.

L'idéal serait évidemment que chaque type de journaliste, comme c'est le cas pour les séducteurs et les reporters, puisse trouver un quotidien qui partage ses aspirations. Mais la presse d'opinion, que prônent sans le dire les éducateurs, s'envisage difficilement dans le cas des mass media comme on les conçoit en Amérique du Nord. Le sociologue George Herbert Mead notait il y a déjà plus de 50 ans qu'il y avait deux grands modèles de journaux en Amérique: l'un axé sur l'information (les contenus) et l'autre, sur la façon de dire (le «story model»). ${ }^{20}$

20. Mead, George Herbert, "The nature of aesthetic experience », International Journal of ethics, 36, Juillet 1926. 
Au Québec, les quotidiens du groupe Québécor occupent fort bien ce dernier créneau: ils informent, mais se soucient d'abord de plaire. Par contre, la presse quotidienne d'information, la presse traditionnelle, pourrait bien trouver sa voie en mettant l'accent, comme le souhaitent les enquêteurs-analystes, sur un journalisme de recherche, analytique, rigoureux et documenté mais qui se soucie en même temps du style, qui fasse preuve d'imagination, voire qui séduise. Un journaliste peut vouloir d'abord informer (c'est ce qui distingue l'enquêteur du séducteur, et la presse d'information de la presse populaire) mais il n'arrivera pas à le faire s'il ne cherche aussi à intéresser.

S'il appartient aux patrons des quotidiens d'information de donner aux journalistes les outils (temps, moyens financiers, etc.) leur permettant de pratiquer un journalisme plus original, les artisans devront aussi faire leur bout de chemin. Les carences des entreprises ont peut-être trop souvent servi d'excuse à l'absence d'effort et d'audace, à l'esprit de routine qui caractérise certaines salles de rédaction. En fait, il s'agit de savoir si la volonté réelle de pratiquer un journalisme plus actif, mais aussi plus exigeant, existe au-delà du discours des enquêteurs-analystes que révèlent les données du sondage. 